\title{
Rapport préliminaire sur les travaux de la mission archéologique de Zeyve Höyük-Porsuk 2014
}

Dominique Beyer, Can Karavul, Françoise Laroche-Traunecker et Aksel Tibet

\section{OpenEdition \\ 1 Journals}

\section{Édition électronique}

URL : http://journals.openedition.org/anatoliaantiqua/362

DOI : 10.4000/anatoliaantiqua.362

\section{Éditeur}

IFEA

Édition imprimée

Date de publication : 1 juin 2015

Pagination : 275-290

ISBN : 9782362450600

ISSN : 1018-1946

\section{Référence électronique}

Dominique Beyer, Can Karavul, Françoise Laroche-Traunecker et Aksel Tibet, « Rapport préliminaire sur les travaux de la mission archéologique de Zeyve Höyük-Porsuk 2014 », Anatolia Antiqua [En ligne], XXIII | 2015, mis en ligne le 30 juin 2018, consulté le 18 décembre 2020. URL : http://

journals.openedition.org/anatoliaantiqua/362 ; DOI : https://doi.org/10.4000/anatoliaantiqua.362 


\title{
ANATOLIA ANTIQUA ESKI ANADOLU
}

\author{
XXIII
}

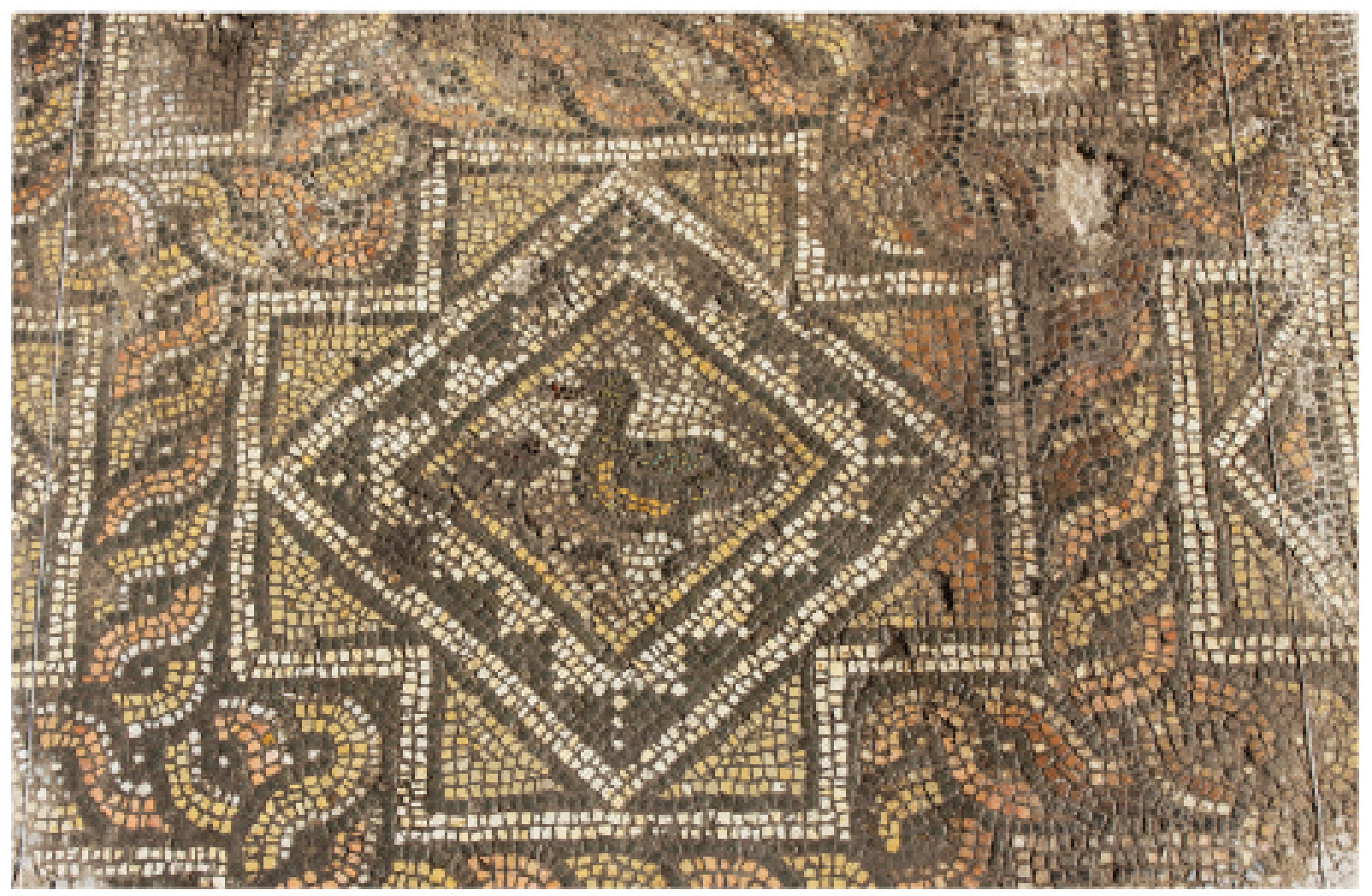

INSTITUT FRANC̣AIS D'ETUDES ANATOLIENNES GEORGES-DUMEZIL CNRS USR 3131 


\section{TABLE DES MATIERES}

Hélène BOUILLON,

On the anatolian origins of some Late Bronze egyptian vessel forms

Agneta FRECCERO,

Marble trade in Antiquity. Looking at Labraunda

Şehnaz ERASLAN,

Dionysus and Ariadne in the light of Antiocheia and Zeugma Mosaics

Ergün LAFLI et Gülseren KAN ŞAHIN,

Middle Byzantine ceramics from Southwestern Paphlagonia

Mustafa AKASLAN, Doğan DEMIRCİ et Özgür PERÇİN en collaboration avec Guy LABARRE, L'église paléochrétienne de Bindeos (Pisidie)

Anaïs LAMESA,

La chapelle des Donateurs à Soğanlı, nouvelle fondation de la famille des Sképidès

Martine ASSENAT et Antoine PEREZ,

Localisation et chronologie des moulins hydrauliques d'Amida. A propos d'Ammien Marcellin,

XVIII, 8,11

Helke KAMMERER-GROTHAUS,

$»$ Ubi Troia fuit«

Atzik-Köy - Eine Theorie von Heinrich Nikolaus Ulrichs (1843)

CHRONIQUES DES TRAVAUX ARCHEOLOGIQUES EN TURQUIE, 2015

Sami PATACI et Ergün LAFLI,

Surveys in Ardahan on the turkish-georgian borderline in 2013 and 2014

Çĭ̆dem MANER,

Preliminary report on the second season of the Konya-Ereğli survey (KEYAR) 2014

Dominique BEYER, Can KARAVUL, Françoise LAROCHE-TRAUNECKER et Aksel TiBBET,

Rapport préliminaire sur les travaux de la mission archéologique de Zeyve Höyük-Porsuk 2014

Jean-Charles MORETTI avec la collaboration de Nicolas BRESCH, Isabel BONORA,

Jean-Jacques MALMARY et Olivier RISS,

Claros, le temple d'Apollon : travaux réalisés en 2014

Olivier HENRY et Erika ANDERSSON, Christophe BOST, Ömür Dünya ÇAKMAKLI, Angela COMMITO, Mélissa CORMIER-HUGUET, Peter DE STAEBLER, Pierre DUPONT, Duygu ERGENÇ, Axel FREJMAN, Banu KEPENEK, Pascal LEBOUTEILLER, Haral NILSSON, Felipe ROJAS, Baptiste VERGNAUD, 


\author{
Dominique BEYER* ${ }^{*}$ Can KARAVUL ${ }^{* *}$, \\ Françoise LAROCHE-TRAUNECKER* ${ }^{*}$ et Aksel TIBBET ${ }^{* * *}$
}

\title{
RAPPORT PRELIMINAIRE SUR LES TRAVAUX DE LA MISSION ARCHEOLOGIQUE DE ZEYVE HÖYÜK-PORSUK 2014
}

\begin{abstract}
La mission 2014 a eu lieu entre le 21 juillet et le 20 septembre. Elle s'était imposée plusieurs objectifs, liés en particulier aux exigences exprimées par les autorités archéologiques d'Ankara au mois de février. En raison de la faiblesse de nos crédits de cette année ${ }^{1}$, nos ambitions en matière de fouille proprement dite ont été très réduites, avec une équipe très réduite également. Mais il était prévu que cette année serait l'année de la prospection géophysique du site, avec la collaboration d'une équipe turque. Cet objectif a été partiellement atteint. D'autre part, les efforts ont porté sur la sécurisation des chantiers et la mise en valeur du site. Une partie du temps disponible a été consacrée également, comme l'an dernier, aux travaux de préparation aux publications.
\end{abstract}

\section{LES OPERATIONS DE FOUILLE}

Grâce à la compréhension de notre représentant des antiquités, Mustafa Poyraz, temsilci envoyé par la Direction générale d'Ankara, nous avons pu reprendre, même partiellement, les sondages interrompus l'an dernier à cause de l'attitude rigide de son prédécesseur de $2013^{2}$.

Ils ont concerné le système des fortifications du chantier II Sud, dans la partie ouest du site et, malgré leur caractère limité, ils ont apporté des informations importantes.

\section{Travaux sur la façade externe de la tour du Bronze (carré J04)}

La tour de plan rectangulaire mise au jour sur le sommet de la butte ouest du site est une des découvertes récentes les plus intéressantes (Fig. 1). Son plan, avec deux espaces internes parallèles, allongés dans le sens de l'axe des courtines, à cheval sur le rempart (pour le niveau supérieur), est exceptionnel au sein de la documentation anatolienne de l'Age $\mathrm{du}$ Bronze. On ne peut guère rapprocher ce parti que de celui des tours du Poternenmaner de la capitale hittite de Boğazköy, mais avec des différences notables. Recouverte par les vestiges très dégradés des remparts de pierres du Fer Moyen $\left(8^{\mathrm{e}}\right.$ siècle av. J.-C., Porsuk III), cette tour bénéficie d'une conservation assez étonnante, grâce, paradoxalement, aux incendies dont elle a été victime à plusieurs reprises.

Le niveau supérieur, le mieux connu, avec sol intérieur $(\Delta 116,59)$, murs de briques (cuites par l'incendie) sur socle de pierres, appartient à la phase Porsuk V, aux environs des $16^{\mathrm{e}}-15^{\mathrm{e}}$ siècles av. J.-C., même si aucun matériel clairement datable n'y a été découvert. Ce niveau correspond, comme ailleurs, à la reconstruction, mais sur un plan semble-t-il différent, de la tour du niveau inférieur (Porsuk VI), qui devrait appartenir à la première phase du dispositif des fortifications, durant la seconde moitié du $17^{\mathrm{e}}$

*) UMR 7044 ARCHIMEDE CNRS-Université de Strasbourg.

**) Université de Sakarya.

***) IFEA-USR 3131, Istanbul.

1) Précisons ici que, malgré nos efforts, nous n'avons toujours pas de sponsors, difficiles à trouver pour ce type d'archéologie d'une part, peu spectaculaire aux yeux du grand public, et pour cette région peu fréquentée par le tourisme d'autre part, la Cappadoce touristique s'étendant une centaine de km plus au Nord.

L'essentiel de nos crédits provenait, comme à l'accoutumée, du Ministère français des Affaires Etrangères et Européennes, que nous remercions vivement. Nous avons également pu bénéficier de l'aide de l'UMR strasbourgeoise 7044 ARCHIMEDE. Le directeur de l'IFEA nous a, une nouvelle fois, prêté gracieusement l'indispensable et fidèle véhicule Ford Transit, sans lequel rien n'est possible sur le terrain.

L'accueil du musée de Niğde et de son personnel a été comme toujours chaleureux et efficace.

2) Cf. le rapport paru dans Anatolia Antiqua XXII (2014) : 327-342. 

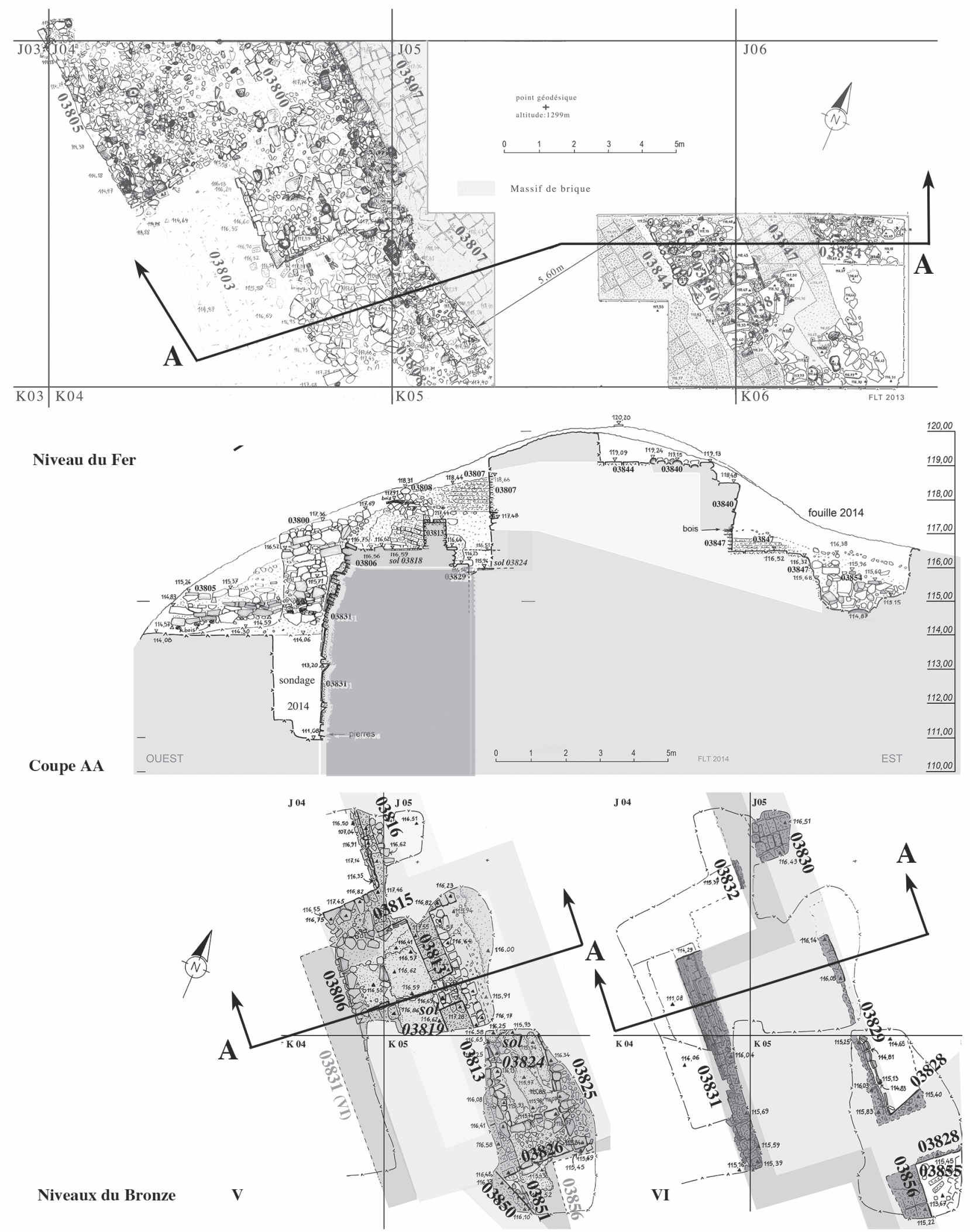

Fig. 1 : Chantier II Sud. Vestiges des remparts du Fer (pierres puis briques de Porsuk III) au-dessus de la tour rectangulaire du Bronze (plans schématiques des phases V et VI). 
siècle av. J.-C. Il convenait de tenter d'en retrouver la base, le long de la façade externe, mais aussi dans un sondage immédiatement au Sud-Est de la tour (cf. infra).

La façade externe de la tour, dont plusieurs mètres de hauteur avaient déjà été dégagés l'an dernier, avait été soigneusement protégée à la fin de la campagne précédente par un toit de tôle, la mettant à l'abri des puissants vents en provenance du plateau anatolien. C'est à l'intérieur de cet espace protégé qu'une tranchée a été établie durant cette campagne (L. 2, $80 \mathrm{~m} ; 1.1,30 \mathrm{~m}$ ) pour suivre, au moins dans la partie nord, l'élévation du mur (03831) en profondeur. Auparavant, l'examen en surface du parement externe du mur, en en enlevant certains éléments ce qui nous était interdit l'an dernier -, montre que ce que l'on considérait précédemment comme des traces d'enduit de réfection sont en fait, au moins ponctuellement, dans la partie supérieure du parement, les restes de joints verticaux dans l'appareil des briques, une grande partie de ces briques étant tombée lors de la destruction, révélant parfois la présence ponctuelle de petites plaques de grès. Ceci ne veut pas dire que des remaniements ne sont pas visibles, au contraire les exemples se sont multipliés d'indices de 'replâtrage' à l'aide d'enduit d'argile, dissimulant une structure interne souvent très bousculée, dont on pouvait se demander parfois comment elle avait pu tenir, avec ponctuellement une brique presque de chant intégrée dans le parement, pour en soutenir vraisemblablement d'autres (Fig. 2). C'est dans la partie inférieure, plus régulière dans sa verticalité (cf. la coupe AA de la Fig. 1) que les zones d'enduit ont été retrouvées en place, même à l'angle nord-ouest, pourtant très exposé (Fig. 3), l'enduit dissimulant l'appareil des briques dont la hauteur était généralement de 10-11 cm, soit légèrement inférieure à celle des briques du niveau V $(12-13 \mathrm{~cm})$. Là où les briques sont visibles, et surtout dans la partie supérieure du mur, on constate qu'elles versent vers l'intérieur de la tour, ce qui s'accorderait assez bien avec une épaisseur du mur relativement faible et la présence d'un espace interne, salle étroite et allongée (?), comme la fouille a permis de le constater pour le niveau V, juste au-dessus. Plus bas, on constate ponctuellement des zones de calcination blanchâtre, poudreuse, derrière l'enduit rougeâtre cuit par l'incendie. On s'attendait à y retrouver les traces d'un chaînage de bois, même rudimentaire, qui aurait entièrement brûlé, mais rien de clair à cet égard n'a pu être observé. Sans doute aurait-il fallu enlever tous les restes d'enduit pour le vérifier, mais cela ne nous était pas possible. Ailleurs, c'est la dureté des restes calcinés contre l'enduit qui a été particulièrement remarquable, empêchant une mise à nu complète du parement.

En raison des difficultés de cette fouille en profondeur, l'espace de la tranchée a été durant l'opération limité à la moitié est, contigüe à la face du mur. C'est là que sous env. 4,50 $\mathrm{m}$ de superstructure de briques (pour le seul niveau VI), sont apparues les premières pierres $(\Delta 112,10)$, sortes de grosses plaques de grès grisâtre en deux couches, sur 30 à $40 \mathrm{~cm}$ de hauteur dégagée, du soubassement habituel de pierres (Fig. 4). Ces plaques étaient encore partiellement recouvertes de l'enduit de terre.

Il devenait toutefois trop difficile et trop dangereux de poursuivre en profondeur : la base de la tour et le sol du niveau VI nous échappent à nouveau, malgré nos efforts.

Il est difficile d'évaluer la hauteur d'origine du soubassement de pierres : dans le cas de la tour nord-est du même chantier II, dégagée précédemment, ce soubassement dépassait largement les deux mètres de hauteur. Mais le contexte topographique y était différent, en bordure directe de la paroi nord, particulièrement raide, du site. Ici, on peut estimer une hauteur plus réduite, de 1,50 à $2 \mathrm{~m}$ peut-être, ce qui ferait tout de même une hauteur conservée de la tour de ce niveau VI de plus de 6 mètres, conservation parfaitement inédite dans le monde anatolien pour cette période du Bronze, surtout s'il faut y rajouter les $1,50 \mathrm{~m}$ env. conservés du niveau $\mathrm{V}$ (cf. section de la Fig. 1).

Aucun matériel archéologique, en dehors d'un petit tesson amorphe, n'a pu être retrouvé lors de ce sondage profond, aucune trace non plus de ces poutres calcinées présentes en si grand nombre ailleurs dans ce chantier, et qui témoignaient pour chacun des deux niveaux, VI puis V, de la violence de l'incendie.

Pour comprendre cette apparente anomalie, il convient à présent d'évoquer la nature des couches traversées.

Ainsi que le montre la (Fig. 5), illustrant la paroi sud de la tranchée, on constate la superposition d'épaisses couches approximativement horizontales. Dans la partie supérieure, sur $2 \mathrm{~m}$ env. de hauteur, sous le remblai du Fer Moyen, on trouve une couche sableuse à galets et gravillons, qui évoque le comblement des caissons des fortifications du chantier IV, à l'Est du site. En-dessous, une couche de $55 \mathrm{~cm}$ env. d'épaisseur, bien plus argileuse, claire, avec peu de galets. Vient ensuite une couche de $80 \mathrm{~cm}$ env., plus sombre et plus humide, très argileuse, avec quelques traînées de gravillons accompagnés de quelques pierres de conglomérat, avec net pendage vers l'extérieur (Ouest). Cette couche reposait à $\Delta$ 

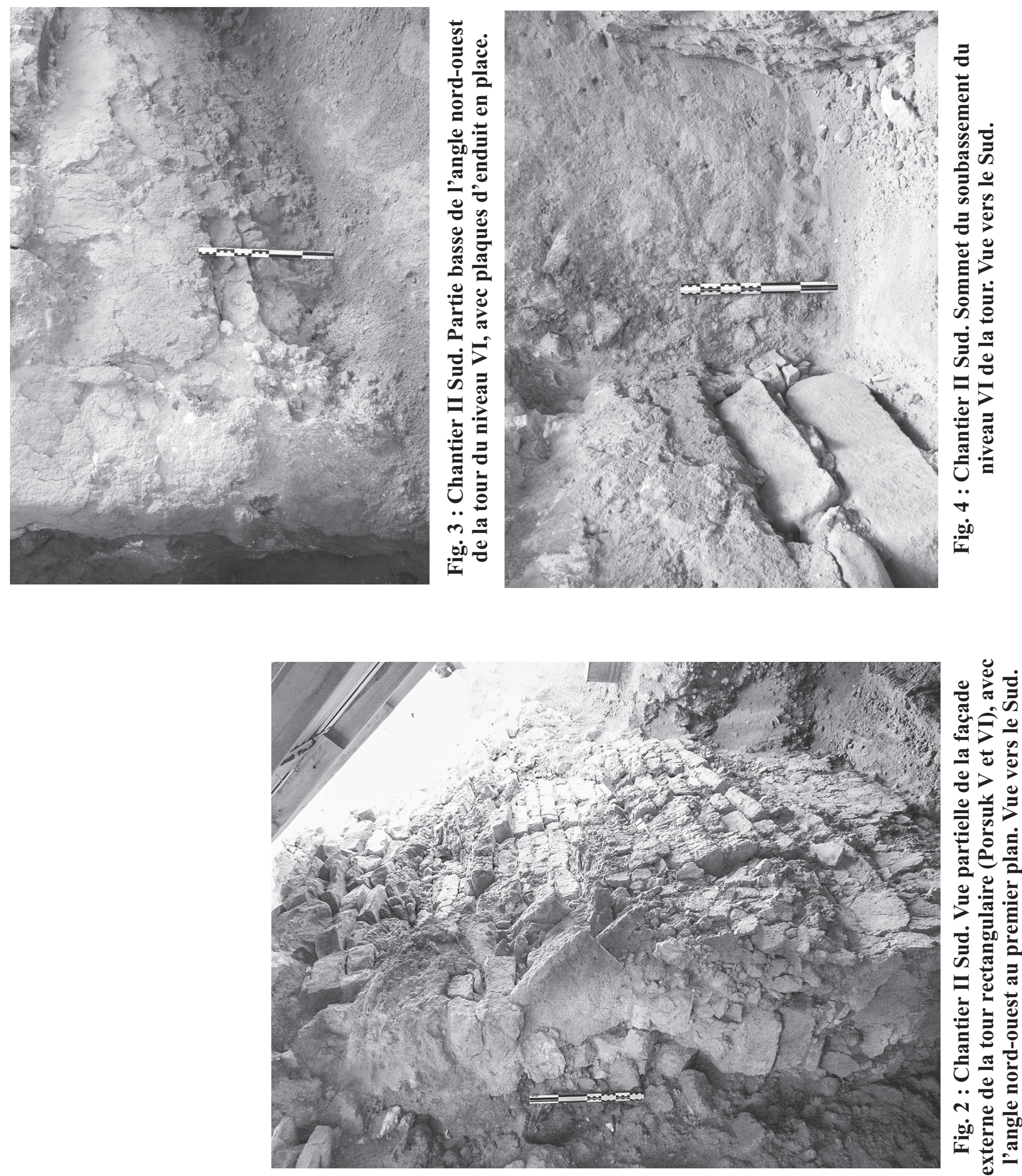


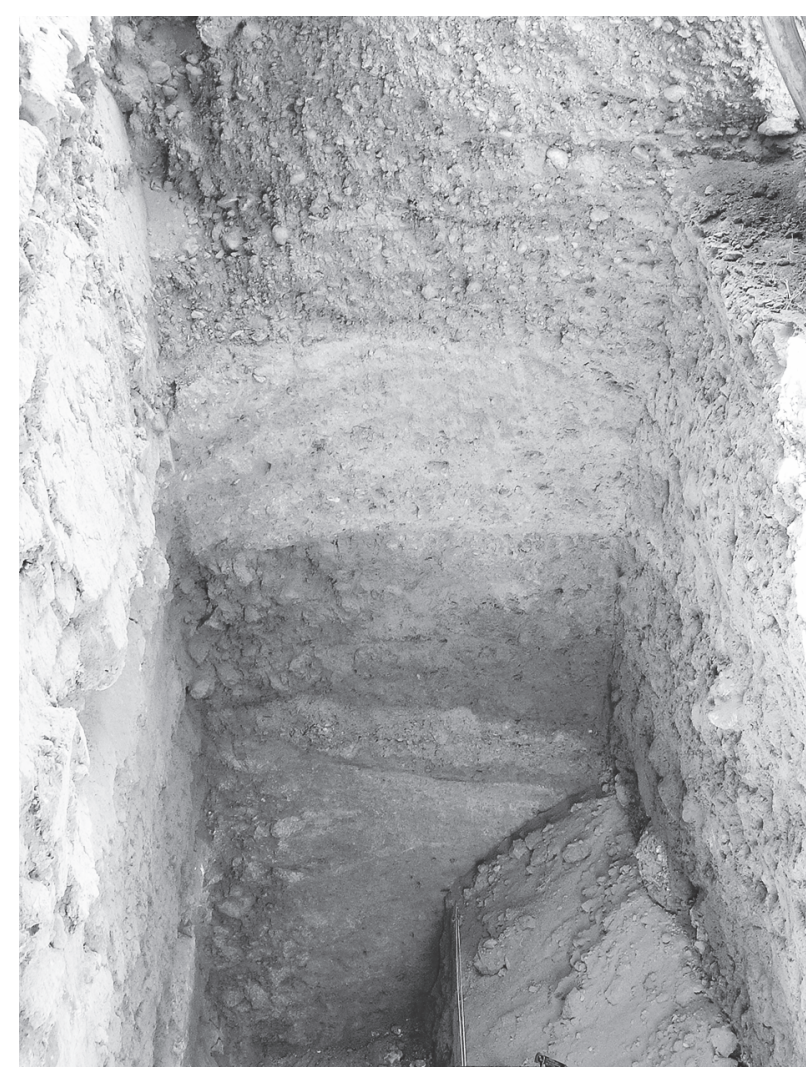

Fig. 5 : Chantier II Sud. Paroi sud de la tranchée, avec les différentes couches de comblement.

112, 60 sur une surface plane, régulière, argileuse tassée, qui évoquait volontiers un sol, au moins un sol de chantier. Ce n'était en fait que le sommet de la couche la plus profonde, dégagée sur une hauteur de $1,20 \mathrm{~m}$, dont le caractère argilo-sableux avec particules blanchâtres (de gypse vraisemblablement) nous rappelait la couche du terrain naturel dans lequel la 'poterne hittite', par exemple, avait été insérée. Etait-il concevable que les bâtisseurs aient ainsi creusé dans le terrain naturel (dit souvent, mais abusivement 'vierge') pour installer, ici, et profondément, les murs de leur tour, des murs qui ont ensuite sévèrement brûlé ? Ce n'était guère vraisemblable. L'explication doit être la suivante : après la destruction par incendie violent du niveau VI, les bâtisseurs du niveau $\mathrm{V}$ ont sans doute comblé tout l'espace avec des terres rapportées, en provenance du site lui-même (ce terrain naturel) et de la rivière voisine (ces couches à galets et gravillons), pour établir une nouvelle base pour la tour nouvelle (Porsuk V) et pour le glacis qui devait l'accompagner.
Si l'on avait pu étendre la fouille vers l'extérieur, on aurait sans doute pu repérer la pente initiale de ce glacis. Notons ici que les habitants du Fer Moyen, au $8^{\mathrm{e}}$ siècle av. J.-C. (Porsuk III), ont fait de même, lorsqu'ils ont construit, en pierres de gypse, leur rempart au-dessus de celui du Bronze, mais plus à l'extérieur, après avoir remblayé les ruines du niveau précédent, comme la mission de 2012 l'a bien montré ${ }^{3}$. Lors du déroulement de la fouille, l'accumulation, contre la face du mur, d'un certain nombre de fragments divers nous avait paru correspondre aux vestiges de la couche de destruction du niveau VI, surtout que la terre y était passablement rougie. Mais après examen plus approfondi, il semble bien que ces fragments n'appartiennent pas à des briques mais à des sortes de nodules sableux durcis ${ }^{4}$ qui avaient roulé contre le mur lors du déversement des terres du comblement.

Parmi les interrogations qui n'ont pas manqué de surgir lors de ces sondages, figure celle-ci : comment expliquer l'importance de l'incendie qui a provoqué la cuisson de toutes ces briques des niveaux du Bronze, dont les murs semblent bien avoir cuit, ou partiellement, à cœur? Certes, il faut tenir compte des éléments d'un chaînage de bois, assez habituel en Anatolie, et en particulier dans les murs de Porsuk. Mais ici, il serait particulièrement discret. Des ouvrages de bois ont sans doute existé au sommet du rempart, pour soutenir par exemple un encorbellement, mais nous n'en avons plus aucune trace. Et était-ce suffisant ? Faut-il imaginer alors d'importants brasiers allumés par les assaillants au moment de l'assaut, ou après la prise des remparts?

\section{Le sondage au Sud-Est de la tour du Bronze (carré K05)}

Dans cet étroit secteur ici aussi nous n'avions pu obtenir l'autorisation, en 2013, de poursuivre les investigations en profondeur. Aussi a-t-on cette fois pu poursuivre les travaux là où la fouille de l'an dernier avait pu mettre en évidence deux états successifs de la courtine du rempart, 03850 et 03851 , qui présentaient un léger décalage dans l'axe sudest (Fig. 1 en bas).

03850 est une portion de mur de briques, connue sur une longueur de $1,50 \mathrm{~m}$ et conservée sur une hauteur de $70 \mathrm{~cm}$ maximum, soit cinq assises de $12 \mathrm{~cm}$ de h. env., avec joints assez épais (1-3 cm). On voit des traces, mais rares, de deux couches

3) Cf. le rapport d'Anatolia Antiqua XXI (2013) : 214-219.

4) Il convient encore de les montrer à un géologue. On précisera que les mêmes particules blanchâtres gypseuses que dans la couche correspondante figurent dans ces modules qui présentent des formes diverses. 
d'enduit sur les faces des briques bien noircies par le feu. 03851 représente une portion de mur de même longueur, mais de hauteur plus réduite (50 $\mathrm{cm}$ ), avec deux assises de pierres de grès, essentiellement, et quelques petites pierres sur lesquelles reposent les briques du mur 03850, mais avec un décalage que l'on s'explique mal, sinon par un changement d'axe de la courtine durant la phase de Porsuk V (cf . Fig. 6).

Le sondage de cette année, malgré son étroitesse, a pu mettre en évidence un nouveau mur de briques (03856) sur lequel s'appuient les pierres du mur 03851. Il correspond cette fois à la courtine du niveau inférieur, Porsuk VI, mais décalée vers l'Est, dans le prolongement plus ou moins du mur 03829 de la tour (du même niveau, sans que l'on puisse connaître son épaisseur). En revanche, son élévation a pu être étudiée sur 1,60 $\mathrm{m}$ de profondeur, avec des assises de 10 à $11 \mathrm{~cm}$ d'épaisseur (briques de $60 \mathrm{x}$ $32 \mathrm{~cm}$; un module de 48 x $48 \mathrm{~cm}$ a été également retrouvé dans la couche de destruction) et quelques éléments d'un chaînage de bois dont on retrouve les logements, sans trace de bois carbonisé, mais avec de la poussière de briques calcinées : logement d'une longrine (L. $145 \mathrm{~cm}$ ou plus ; d. entre 10 et 14 $\mathrm{cm})$ rejoignant celui d'une boutisse (L. $70 \mathrm{~cm}$ ?; d. max. $20 \mathrm{~cm}$ ), à une cinquantaine de centimètres de l'angle que ce mur forme avec une nouvelle portion de mur de briques, qui doit logiquement correspondre au parement sud-est du mur 03828, bien que ce mur, de la phase Porsuk VI, montrait en 2012 un axe légèrement différent. Il est difficile d'en savoir davantage, car il faudrait alors démonter les restes du mur 03826 qui le surmonte ainsi que le mur 03825, ces deux murs formant l'angle sud-est de la tour rectangulaire de Porsuk V.

Dans ce sondage devenu très étroit en raison de la découverte du mur 03856, la couche de destruction du niveau VI présentait un amoncellement de briques éboulées, fortement cuites par l'incendie, assez impressionnant par sa densité et par sa dureté, raison supplémentaire pour laquelle le sondage a été ici aussi interrompu sans possibilité d'atteindre la base du dispositif.

Malgré ces difficultés, les travaux autour de la tour du chantier II Sud ont confirmé depuis trois campagnes l'importance du site dans le domaine de l'architecture militaire anatolienne, aussi bien pour le Bronze que pour le Fer. Elle montre d'une part des dispositifs originaux et plutôt rares, et d'autre part ces dispositifs, au moins pour la période du Bronze, Moyen et Récent, sont conservés de manière exceptionnelle, ce qui pourrait autoriser dans l'avenir des dégagements spectaculaires, pour autant que soient possibles les indispensables travaux de protection et de mise en valeur.

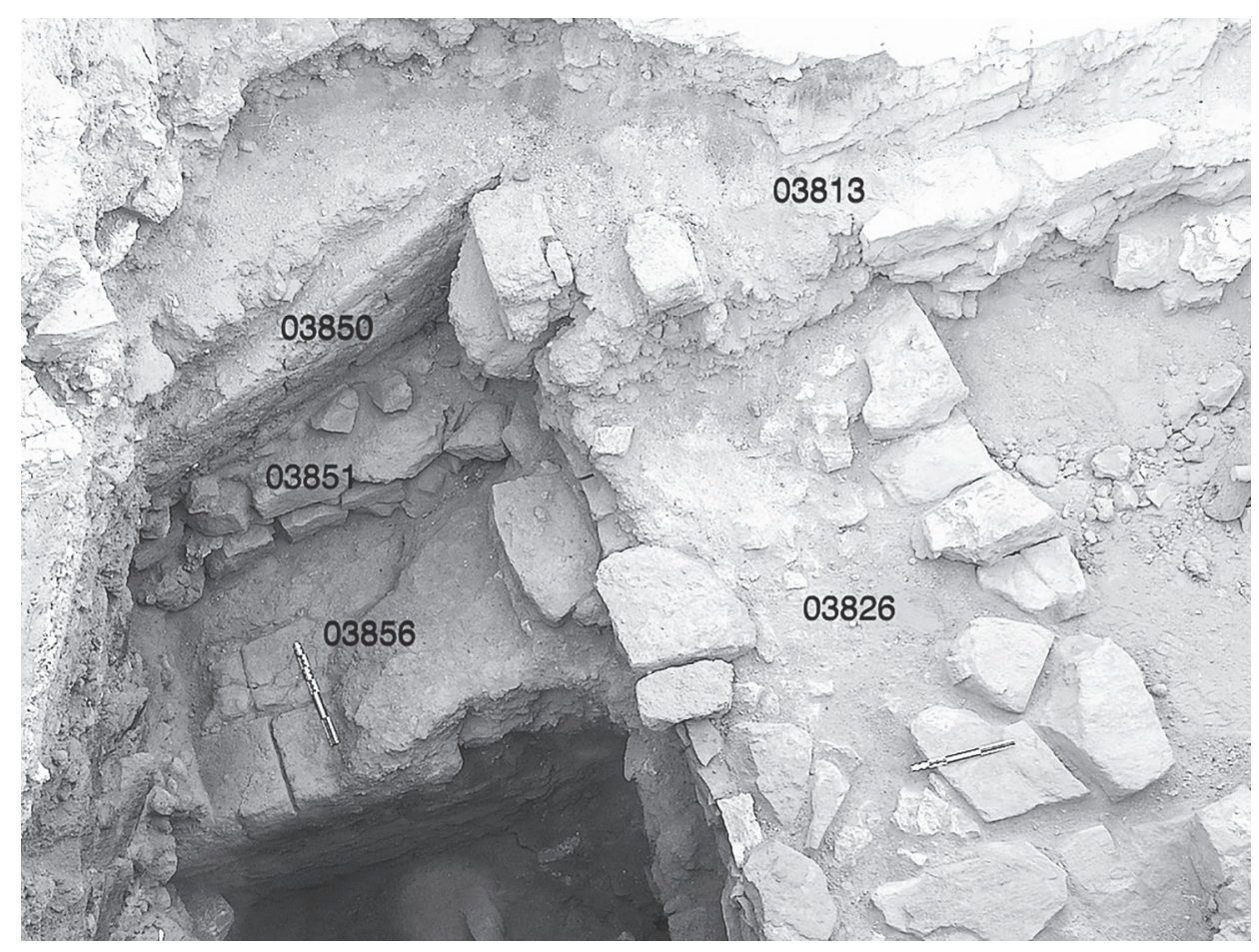

Fig. 6 : Chantier Sud. Le sondage au Sud-Est de la tour du Bronze. Croisement des murs des phases V et VI. Vue prise vers l'Ouest. 


\section{Le sondage en J06 SO}

Dans ce secteur J06 également, qui avait fait l'objet de dégagements l'an dernier, les objectifs initiaux n'avaient pu être réalisés en raison des blocages du temsilci. Il s'agissait, sur la pente est de la butte principale du site, de rechercher les limites du grand massif de briques crues (03807) retrouvé durant les campagnes précédentes sur le sommet de la butte et à proximité du secteur de la tour du Bronze citée plus haut. Ce massif semblait avoir des dimensions importantes, et sa position au sommet du site, très pentu et donc très érodé, fait qu'aucune couche archéologique n'avait pu être mise en relation avec cette construction, par conséquent difficile à dater. Le plan et la section de la Fig. 1 montrent que ce massif s'est implanté profondément dans les vestiges des fortifications du Fer Moyen (Porsuk III, $8^{\mathrm{e}} \mathrm{s}$. av. J.-C.) à l'Ouest (en J04 E et J05 O). Dans les carrés $\mathrm{J} 05 \mathrm{SE}$ et $\mathrm{J} 06 \mathrm{SO}$, les briques grises à particules de gypse, caractéristiques de ce massif ont été retrouvées (= ici 03844).

Mais du côté est, dans la pente, ce massif $(03807=03844)$ a été coupé par un nouveau mur, cette fois de pierres (03840), suivant à peu près le même axe, soit Nord-Ouest/Sud-Est, avec un puissant appareil de blocs de gypse sur le parement nord-est dont nous avons complété le dégagement cette année (Fig. 7-8). Ces blocs reposaient sur une sorte de radier de petites pierres entremêlées de minces boutisses de bois. La technique de construction ainsi que la présence de quelques tessons peints caractéristiques indiqueraient la période hellénistique.

En-dessous, il avait été possible de retrouver des briques (ici 03847), souvent très lessivées, qui paraissaient pouvoir appartenir à notre massif 03844 . Mais un vaste éboulis de pierres les avait recouvertes, que nous n'avons pas eu l'autorisation, l'an dernier, de toucher. Cette couche d'éboulis (03846) provenait certainement de l'effondrement des superstructures du gros mur de pierres (03840) et d'une sorte de contrefort qui l'accompagnait (03841). C'est cette masse de pierres que nous avons voulu cette année explorer pour tenter de retrouver les limites orientales du grand massif de briques grises et de préciser sa nature. Le long de la paroi nord de la partie méridionale de J06, une tranchée a ainsi été établie, large de $1,50 \mathrm{~m}$, dont le but était de pouvoir étudier la stratigraphie en coupant dans toutes ces couches (Fig. 8).

Dans la partie ouest, l'appareil des briques du mur 03847 a bien pu être mis en évidence, alors qu'au Sud du carré, les briques sont trop lessivées sous les éboulis. La fouille a pu repérer, au centre de la tranchée, la limite nord-est du mur, très dégradée, et que l'on a pu suivre, en profondeur, sur $1,50 \mathrm{~m}$ env. jusqu'à la cote $\Delta 115$. Ici non plus, la base n'a pu être atteinte, la fouille ayant été suspendue à $\Delta 114,80$, en raison cette fois de la densité trop forte des éboulis

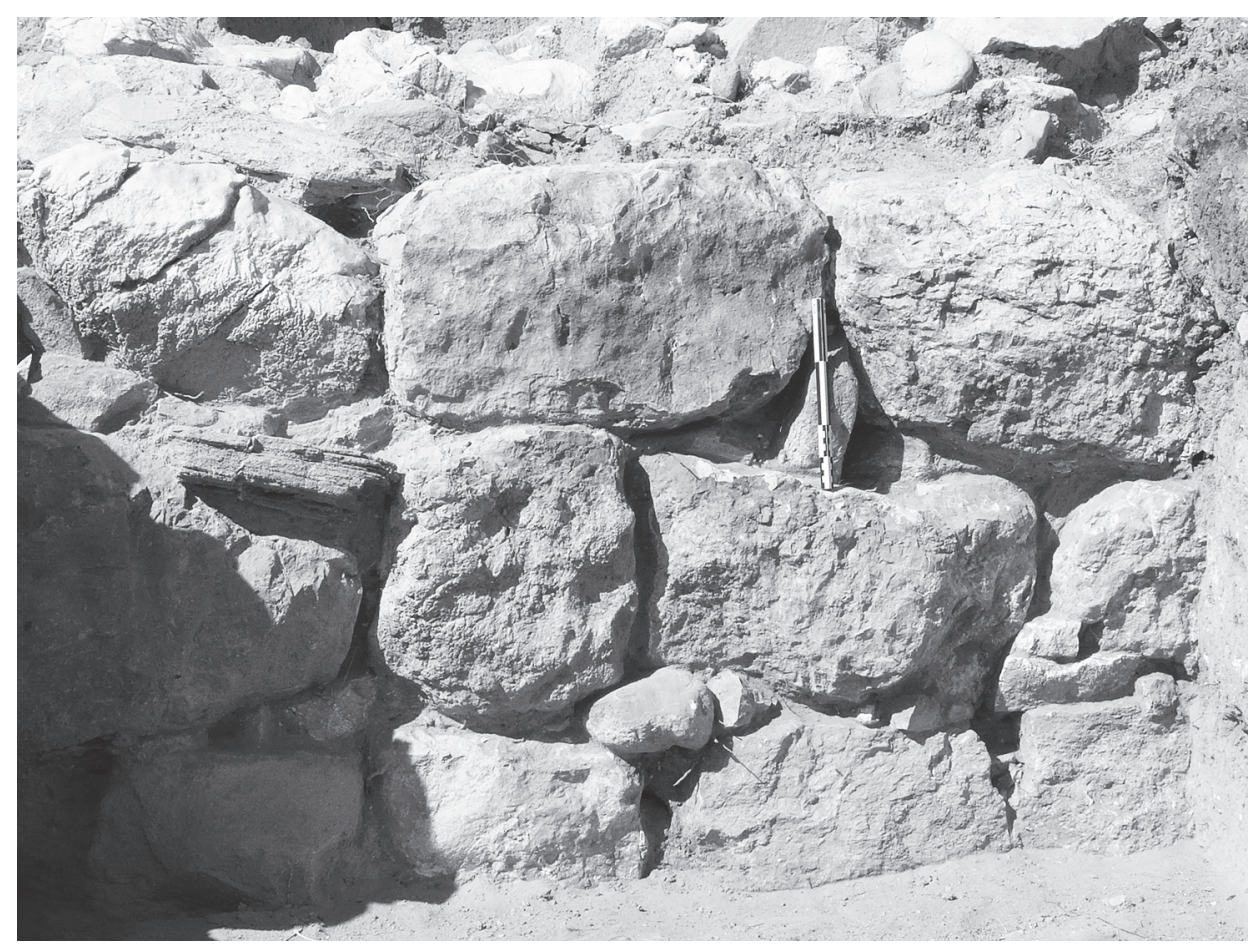

Fig. 7 : Chantier II Sud. Le sondage en J06 SO : élévation du mur de pierres 03840, sans doute hellénistique. Vue vers l'Ouest. 


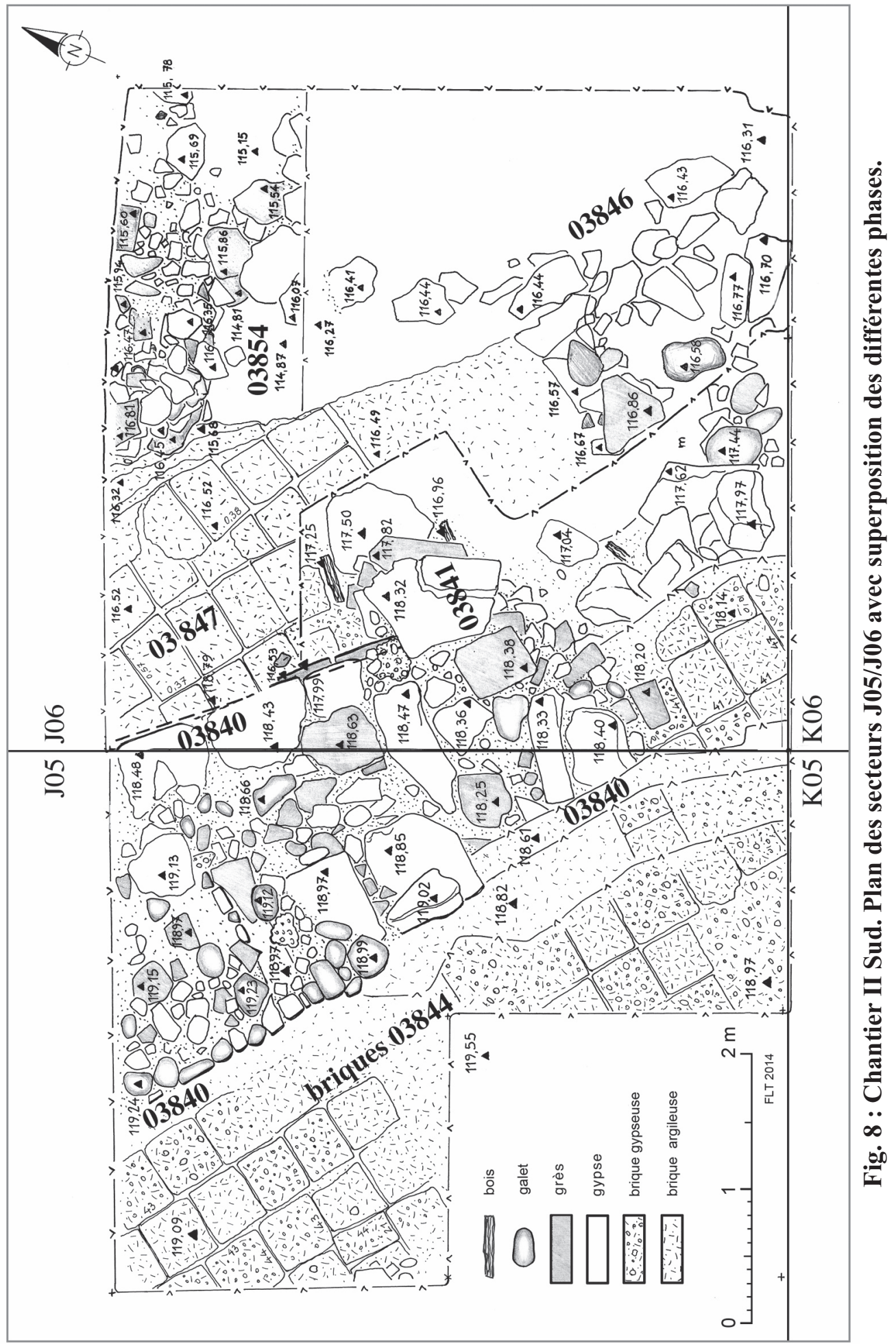


de pierres, gypse et grès mêlés (Fig. 9). A plusieurs reprises, cette masse de pierres (03854) nous a donné l'impression de correspondre aux restes d'un mur appuyé contre les vestiges du mur de briques. Mais en définitive, l'hypothèse d'un vaste éboulis, évoqué plus haut, semble la plus vraisemblable.

La construction de briques grises, pour une fois non brûlées, forme ainsi un ensemble $(03807+03844+03847)$ épais d'environ $10 \mathrm{~m}$, peutêtre davantage, car les limites sud-ouest, en raison de l'érosion de la pente, en J04 et J05, ne sont pas totalement assurées (cf. aussi Fig. 1, en haut). L'érosion a pu rogner la face nord-est du dispositif également. Dans l'état actuel du dégagement, la longueur est de $15 \mathrm{~m}$ env., avec une orientation Nord-Ouest/Sud-Est, et la hauteur maximale conservée de $4 \mathrm{~m}$, peut-être davantage du côté est. A quoi cette construction massive, sans équivalent à Porsuk, sur le point culminant du höyük, peut-elle correspondre ? Sans doute à une puissante terrasse, assise sur les vestiges des fortifications antérieures, et susceptible de supporter un petit fortin ou un autre édifice dominant l'ensemble du site ? La période à laquelle on peut rattacher cette construction n'a pas livré pour l'instant, à Porsuk, de vestiges très significatifs : il s'agit d'une phase récente (Fer Récent) du niveau Porsuk III, si l'on se fonde, à défaut de matériel archéologique s'y rattachant, à une analyse de radiocarbone faite l'an dernier sur des restes de petites boutisses de bois non carbonisé. Cette analyse a fourni une date des environs de 500 av. J.-C. ${ }^{5}$.

\section{L'OPERATION GEOPHYSIQUE}

Depuis quelques années, cette opération sur l'ensemble du site était envisagée. Elle n'avait pu être réalisée pour diverses raisons. Il s'agissait cette fois de réussir, pendant une durée d'un mois, quitte à réduire nos ambitions en matière de fouille proprement dite.

Nous avons ainsi bénéficié de l'aide apportée par le Dr. Can Karavul, de l'Université de Sakarya, de son assistant Samet Yildı et d'une équipe de 5 étudiants. Le travail a débuté dans la partie ouest du site, après débroussaillage, et il devait se poursuivre régulièrement jusqu'à l'est, au chantier IV (Fig. 10). Si l'activité a été intense et solide, en revanche, pris par d'autres obligations professionnelles, le Dr. Karavul et son équipe nous ont quittés au bout d'une semaine, ce qui n'était naturellement pas prévu! L'opération n'est donc que partiellement réussie et elle devra être poursuivie l'an prochain.

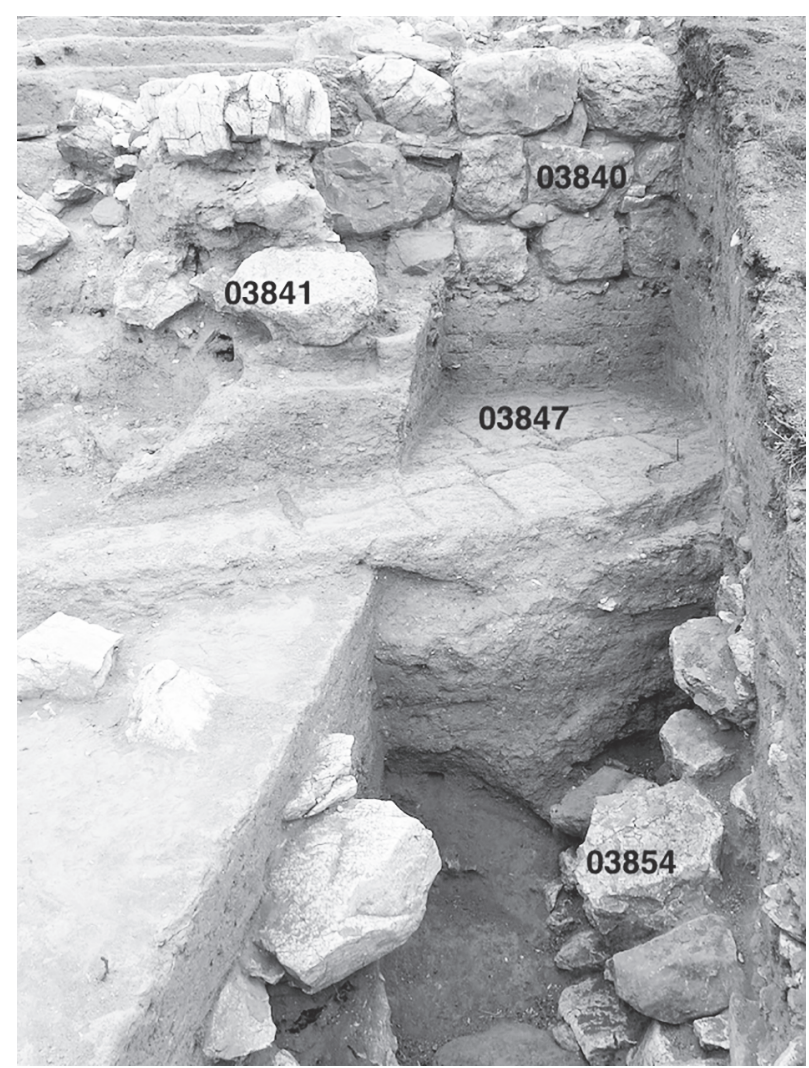

Fig. 9 : Chantier II Sud. Secteur J06 : éboulis dans la tranchée nord, contre les vestiges du mur de briques 03847 . Vue vers l'Ouest.

Le plan fourni ci-après (Fig. 11) est dû à Françoise Laroche-Traunecker qui a intégré les données topographiques des données géophysiques qui nous ont été fournies sur le plan topographique de la partie ouest du site. Un relevé géomagnétique de base, en forme de $\mathrm{L}$ renversé, à l'Est et au Sud-Est de la butte du chantier II où se sont déroulées les opérations de fouilles, a été réalisé dans un premier temps. Il a pu mettre en évidence la présence de plusieurs anomalies circulaires, dont ce qui a été considéré vraisemblablement comme un puits en 73/10. Dans la bande 07/08, les traces d'un bâtiment rectangulaire auraient été repérées, dont la nature est évidemment délicate à préciser. Durant ses travaux sur le terrain, l'équipe de Can Karavul a été d'autre part intriguée par la concentration de deux masses de briques brûlées réagissant très fort, au Sud de la butte, à peu près à l'emplacement de la piste menant, sur la pente, vers le sommet : une étude détaillée y a alors été menée, avec le procédé de résistivité électrique (Fig. 12). Ces deux masses, situées sur la ligne des

5) Analyse pratiquée par le Poznan Radiocarbon Laboratory (Pologne) sur l'échantillon PK 13-03847.0001, provenant des briques de la portion 03847 , en J06. 


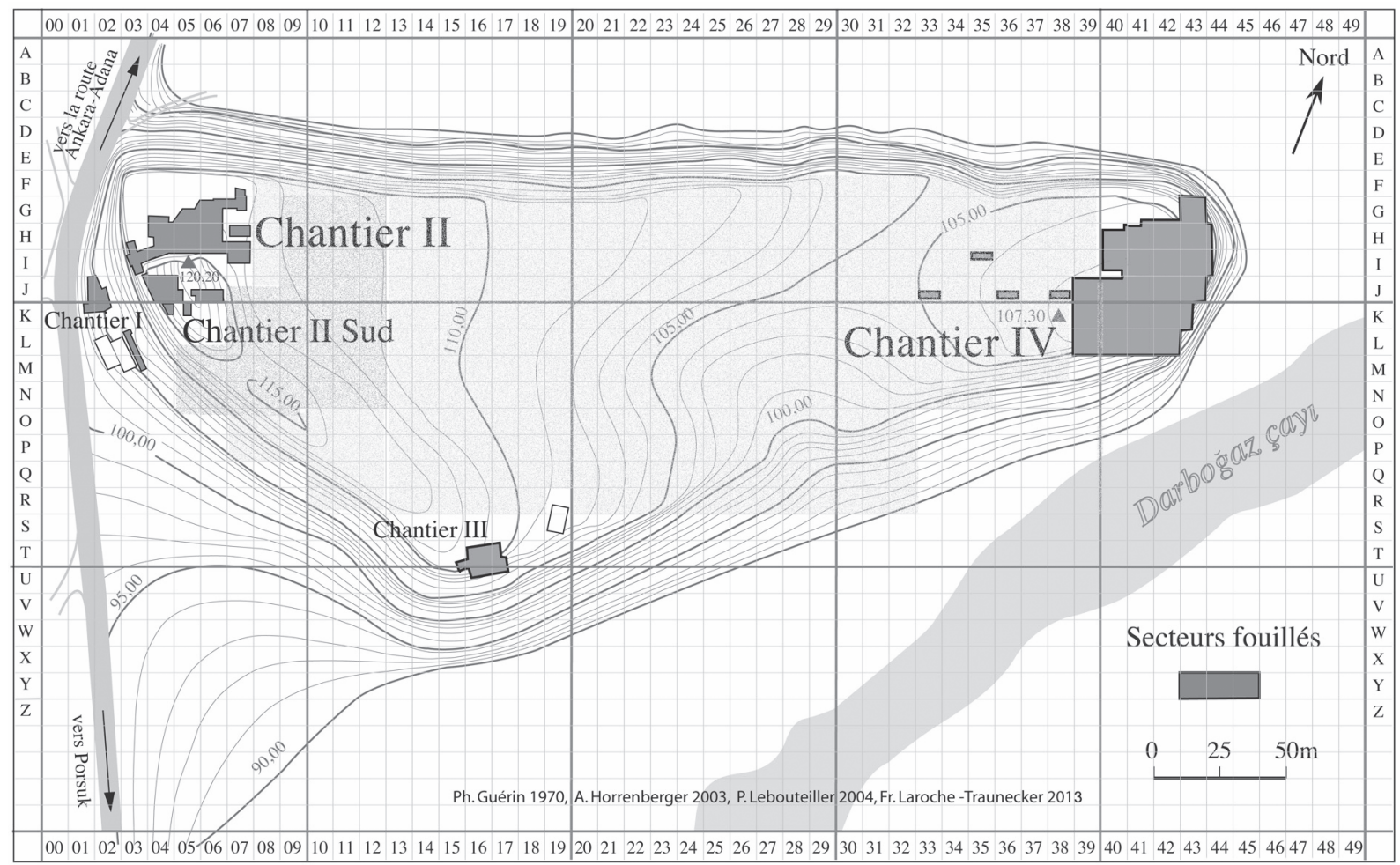

Fig. 10 : Implantation du carroyage pour l'opération géophysique au chantier II Sud.

remparts, pourraient correspondre à des tours, mais la distance assez faible qui les sépare, d'env. $3 \mathrm{~m}$, en 11-13/23-25, indiquerait alors la présence d'une porte de ville. La présence à cet endroit d'une porte de ville paraît assez logique si l'on tient compte du fait que la piste actuelle, donnant accès au sommet du höyük, passe à cet endroit et qu'elle a vraisemblablement depuis longtemps emprunté un tracé antique. La découverte de l'équipe des géophysiciens est à l'évidence intéressante, d'autant que la 'tour' amont semble révéler une densité métallique très forte, inhabituelle, d'après les spécialistes, ce qui ne laisse pas d'intriguer, surtout pour un site dont on connaît les liens avec la métallurgie, mais où nulle part un ensemble métallique n'a pu être mis au jour : dépôt avant exportation vers la capitale hittite, dépôt à caractère militaire, autre ?

\section{LES TRAVAUX DE SECURISATION, RESTAURATION ET MISE EN VALEUR DU SITE}

Sous ce titre nous entendons toute une série d'opérations fortement demandées par les autorités locales depuis quelques années. Nous avions déjà réalisé, régulièrement, ces derniers temps, la consolidation de certains murs, des réaménagements de clôtures, la disposition de deux panneaux pédago- giques, à l'entrée du site et au chantier II. Ces travaux ont été multipliés cette année.

1. Au chantier IV, extrémité orientale du site, les clôtures ont été entièrement revues, partiellement déplacées pour faciliter la visite, et pourvues d'un nouveau grillage. Du côté nord-est, en bordure sécurisée des vestiges d'un sondage profond, on a pu réutiliser un tas de pierres des déblais de fouille pour réaliser une petite plateforme d'observation pour les visiteurs, avec un panneau explicatif trilingue des dégagements opérés dans les couches supérieures de ce vaste secteur (Fig. 13).

2. A l'autre extrémité du site, au chantier II, les clôtures entourant les chantiers ont été également renouvelées et les accès aux zones pouvant présenter un danger pour les visiteurs sécurisés. Un passage particulier, clôturé (Fig. 14), permet dorénavant aux visiteurs d'accéder plus à l'intérieur de ce secteur (partie nord) et de bénéficier d'un panneau explicitant la stratigraphie assez complexe du système des fortifications de ce secteur.

Dans cette zone, les vestiges des murs des fortifications du Fer, en bordure nord du site, ont été consolidés et complétés, à des fins de conservation, sous le contrôle de notre temsilci, par des pierres de gypse, ce qui a permis aussi de rendre l'ensemble plus lisible (Fig. 15). 


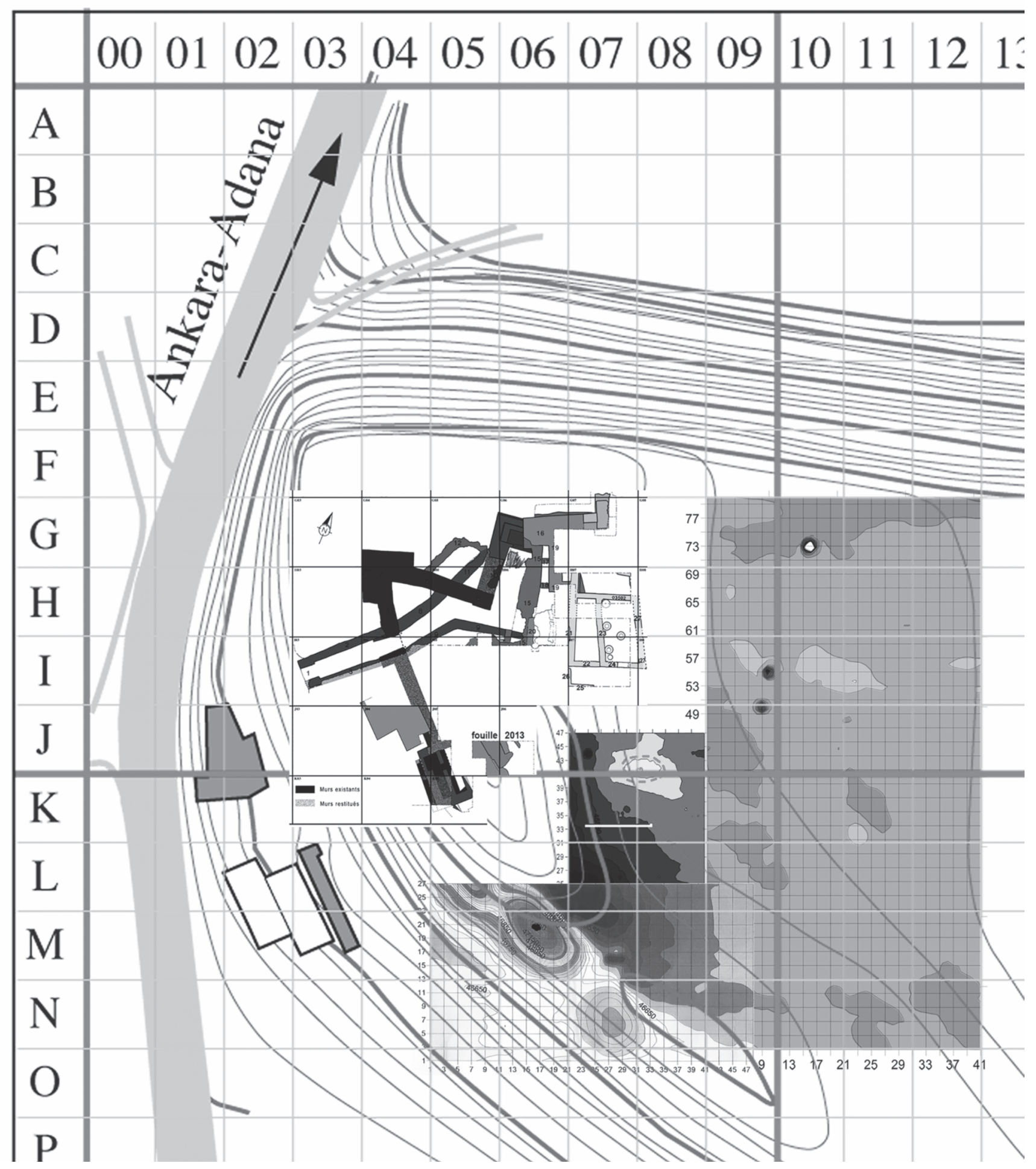

Fig. 11 : Plan de la partie ouest du site (chantier II) avec vestiges archéologiques et implantation des recherches géophysiques révélant les anomalies observées par l'équipe de Can Karavul. 

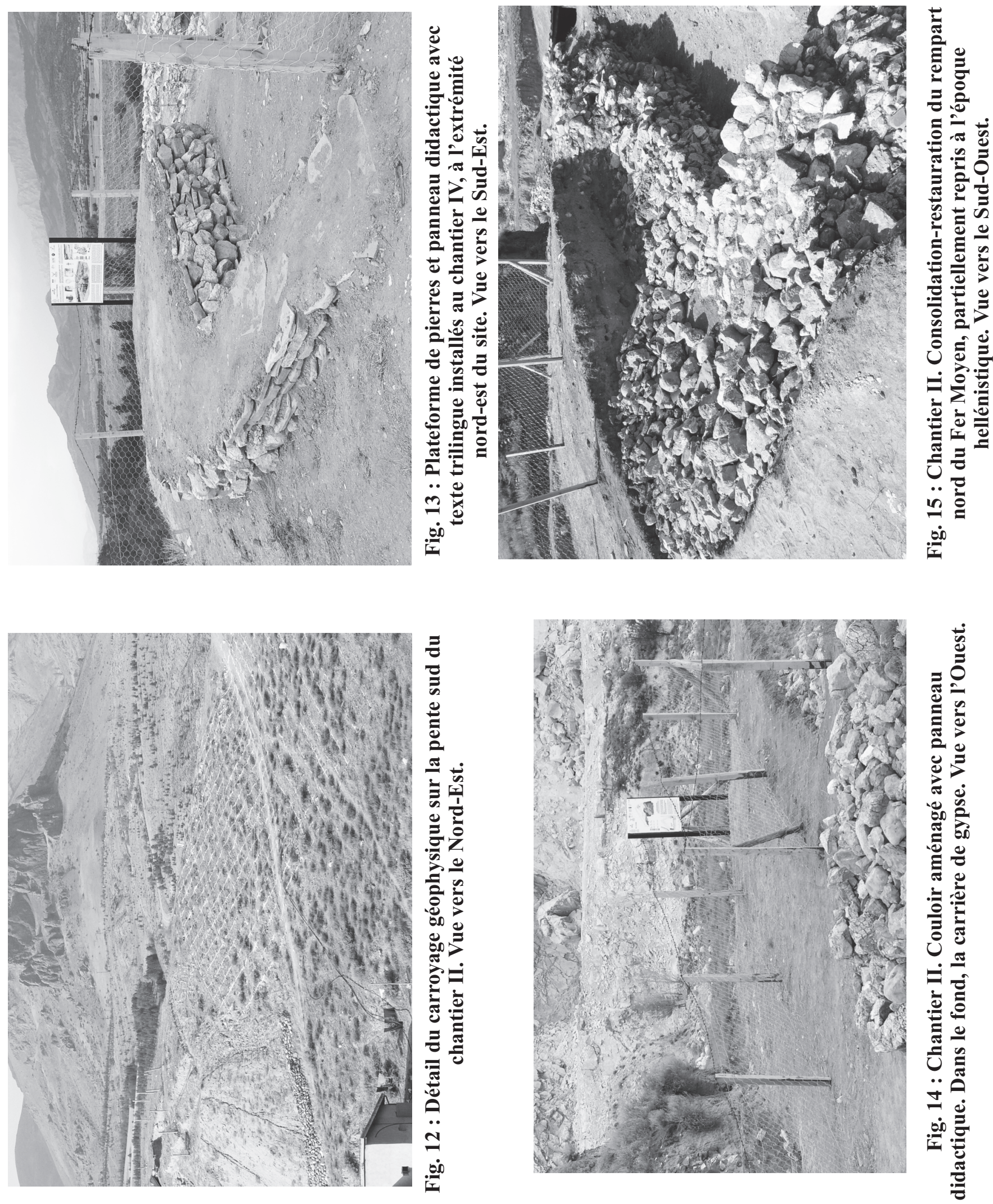
3. La question de la préservation-restauration du secteur de la 'poterne hittite' se pose depuis bien des années et elle n'a pas trouvé jusqu'à présent de réponse satisfaisante. Le projet de couverture de protection présenté il y a quelques années aux autorités turques par la mission n'avait pas été agréé, étant considéré par la commission ad hoc de Nevşehir comme en somme insuffisamment élaboré. Le chantier ayant évolué depuis, avec le dégagement de vestiges similaires à préserver, et nos moyens financiers restant trop limités, nous avons depuis un certain temps envisagé une autre solution, plus simple, plus économique et peut-être plus satisfaisante. Celle-ci consisterait à restaurer et recouvrir les tours et murs de fortification construits en briques (plus ou moins cuites par l'incendie) par un revêtement de briques modernes, mais conçues selon les modules et principes des briques de la période hittite.

Durant cette campagne, nous avons ainsi voulu tester cette solution, non pas sur les vestiges euxmêmes - il aurait fallu pour cela obtenir les autorisations préalables des autorités -, mais en construisant, à proximité de la maison de fouille, une sorte de mur-témoin (Fig. 16) avec, sur socle de pierres, quatre blocs appareillés de briques fabriquées par nos soins avec des compositions variées. Les briques, de $40 \times 18 \times 12-13 \mathrm{~cm}$, ont été réalisées dans des moules en bois à la manière traditionnelle (Fig. 1718). Le mortier était fait sur place, dans une fosse bâchée, de terre du site, en partie des déblais de la fouille des remparts, d'eau et de paille hachée ( 20 $\%$ en volume). C'est ce que contenait le premier bloc. Le second a vu le mélange initial complété avec de la chaux. Dans le troisième, sable et graviers de la rivière ( $30 \%$ env. en volume) ont été ajoutés au mélange initial sans la chaux. Enfin dans le quatrième, à gauche sur la photo de la Fig. 16, fabriqué avec le mélange précédent complété avec de la chaux, ont été tentés des essais de coloration avec diverses proportions pour se rapprocher de la teinte des briques cuites. On précisera ici que le ciment, qui a été utilisé avec relativement de succès dans d'autres contextes (briques d'adobe dans certaines restaurations à Mari, Syrie, par exemple), est banni des chantiers de Turquie par les autorités locales. Le but de ces diverses expérimentations, lors de notre campagne 2014, était double. Il était d'abord technique : tester les capacités de travail de nos ouvriers, selon les méthodes traditionnelles, en adoptant une formule de brique séchée à l'air susceptible de résister le mieux possible aux intempéries, avec surtout la terre du lieu. Le second but était stratégique : trouver une solution satisfaisante de préservation/restauration des murs de briques des fortifications de la période hittite, en proposant dans un premier temps ces expérimentations : le résultat devra être examiné en 2015 sur place, avant de proposer un éventuel plan de restauration aux autorités compétentes.

\section{LA QUESTION DU DEPÔT DU MATERIEL ARCHEOLOGIQUE ET DU LABORATOIRE}

Lors de la réunion d'Ankara, et bien auparavant déjà, les autorités locales avaient souligné le fait que nos conditions de stockage du matériel archéologique, en un petit local dans la maison de fouille, ainsi que dans un dépôt sécurisé sur le site, ne correspondaient pas aux normes actuelles et que ces locaux étaient à l'heure actuelle parfaitement saturés. De plus, nous ne disposons pas jusqu'à présent de véritable laboratoire de restauration digne de ce nom, juste d'un petit espace de premiers secours.

Aussi avions-nous déjà, l'an dernier, exploré les différentes solutions de réutilisation de certains bâtiments administratifs abandonnés des environs (écoles et mairies), suite aux mesures de restructuration récentes, suivant la suggestion du personnel du musée de Niğde. En définitive, la seule solution possible restait celle de l'école abandonnée de l'ancien village d'hiver de Porsuk, de l'autre côté de la grande route nationale (Fig. 19-20). La demande officielle d'attribution de ce bâtiment à la mission a été acceptée par les autorités de l'éducation nationale.

La possibilité nous est alors offerte, moyennant quelques travaux de remise en état, d'utiliser ce bâtiment comme nouveau dépôt de fouille, avec locaux de restauration et, éventuellement, dans l'avenir, aménagement de chambres supplémentaires pour le logement de la mission.

\section{LA DENDROCHRONOLOGIE}

Comme l'an dernier, nous avons accueilli à Porsuk le spécialiste de la dendrochronologie anatolienne, le Pr. Peter Kuniholm, qui avait travaillé depuis bien longtemps avec la mission de Porsuk sur les échantillons nombreux de poutres carbonisées ou non des divers secteurs de la fouille. Porsuk est devenu au cours du temps l'un des sites majeurs d'Anatolie en ce domaine. Actuellement à la retraite, il parraine en quelque sorte les activités d'un nouveau laboratoire américain, celui de l'Université d'Arizona, dirigé par Pr. Charlotte Pearson, avec la collaboration du Pr. Tomasz Wazny, de l'Université polonaise de Torun. Cette collaboration renouvelée nous permet d'envisager de nouvelles analyses d'échantillons 

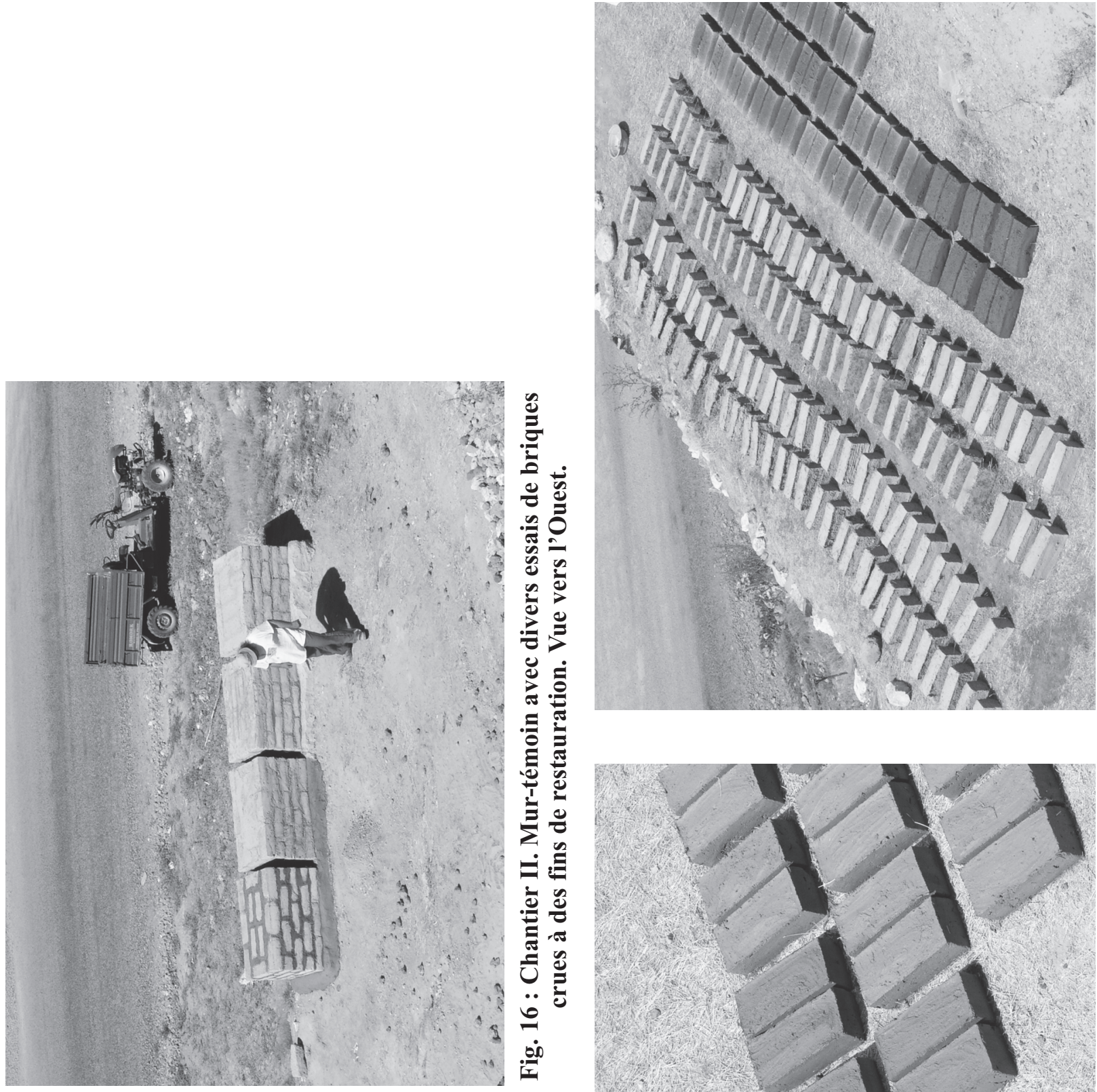

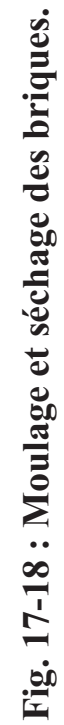

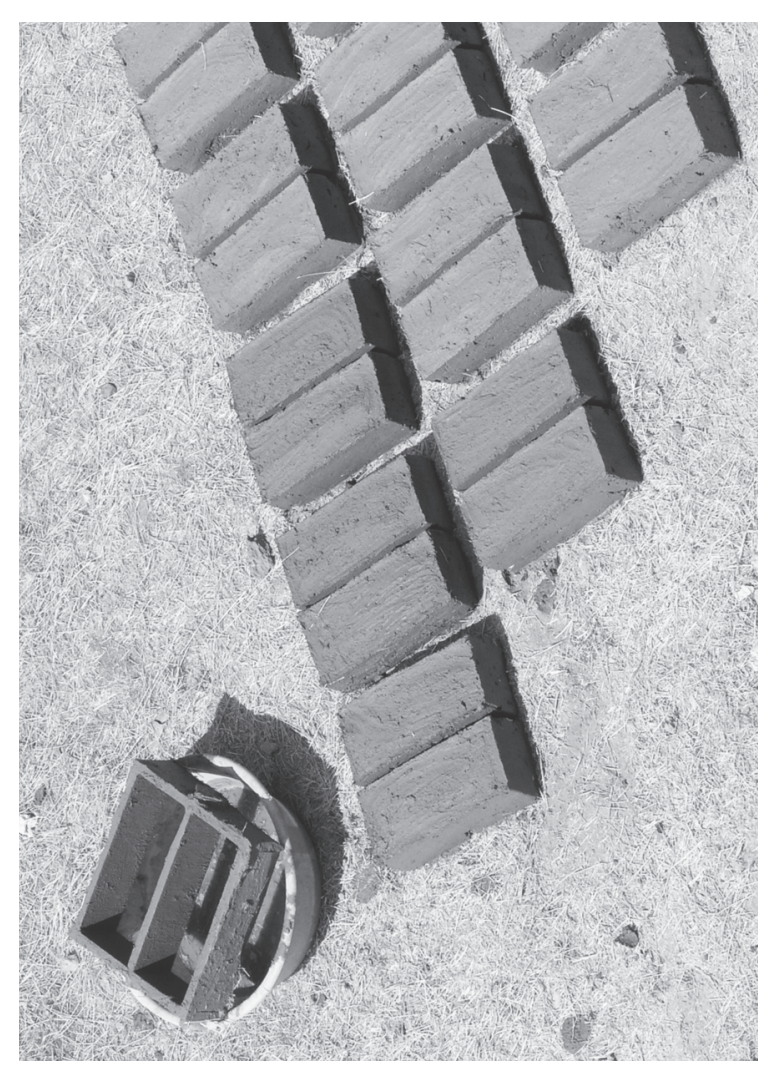



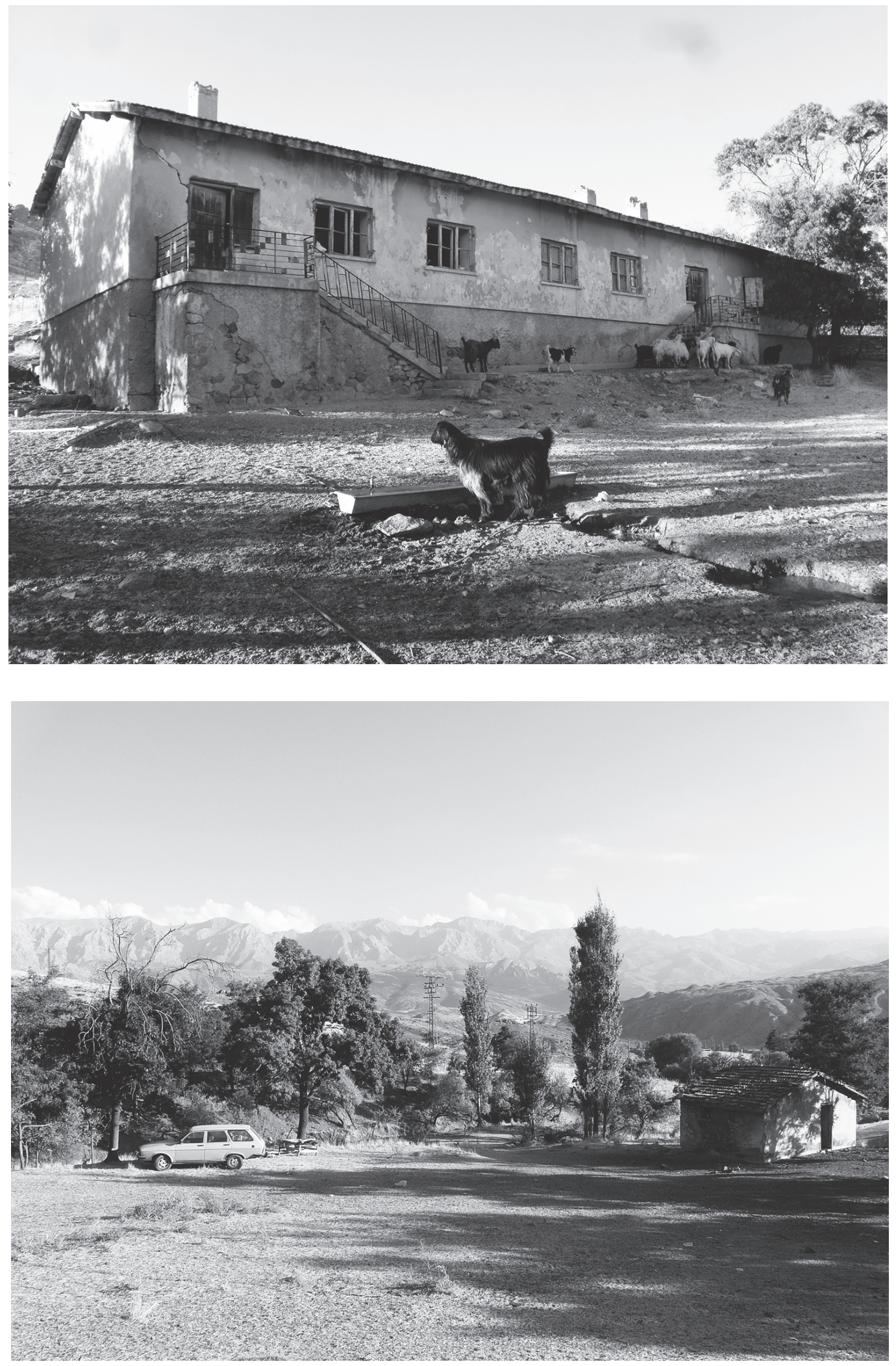

Fig. 19-20 : Vues de l'ancienne école du village d'hiver de Porsuk. 
aussi bien sur des données anciennes à réexaminer, que sur des exemples plus récents. C'est dans ce sens qu'un échantillonnage a pu être exporté vers leur laboratoire.

\section{PUBLICATIONS ET ACTIVITES DE VALORISATION ; EVENEMENTS SCIENTIFIQUES}

Comme l'an dernier, une partie du temps et des forces disponibles en mission a été consacrée aux travaux de la publication, encore en chantier, des résultats des fouilles, anciennes et récentes, dans les niveaux du Bronze et du Fer. Mais se sont greffés sur cette tâche de longue haleine d'autres travaux :

- participation au travail d'édition des actes des Rencontres archéologique de l'IFEA, consacrées, les 8 et 9 novembre 2012, à la Cappadoce méridionale. Porsuk y est présent par plusieurs publications :

Dominique Beyer, "Quelques nouvelles données sur la chronologie des phases anciennes de Porsuk, du Bronze Moyen à la réoccupation du Fer", ainsi que "Préface";

Françoise Kirner, "Fonction des espaces des niveaux hellénistiques et romains du site de Porsuk";

Aksel Tibet et Françoise Laroche-Traunecker, "Les fortifications occidentales de Porsuk, restitution et modélisation des états les plus anciens";

Stéphane Lebreton, "Zeyve-Porsuk : réflexion sur les fouilles des niveaux hellénistiques et romains à partir de la datation de la nécropole"
Julie Patrier, 'Zeyve-Höyük-Porsuk à l'Age du Fer : les fibules, marqueurs chronologiques et d'échanges culturels";

Olivier Pelon $\dagger$, "Le höyük de Porsuk, une forteresse hittite en Cappadoce méridionale".

- essentiel de l'organisation, par les soins de Françoise Laroche-Traunecker et Aksel Tibet, d'une exposition documentaire à la mémoire d'Emmanuel Laroche, éminent hittitologue et ancien directeur de l'IFEA Istanbul, pour son centenaire, accompagnée d'une plaquette. Rappelons ici qu'Emmanuel Laroche est à l'origine de la création de la mission française de Porsuk. Cette exposition a eu lieu en novembre 2014 dans les locaux de l'IFEA en parallèle à un colloque (Rencontres d'archéologie de l'IFEA) sur le même sujet. La mission de Porsuk y a été représentée par les deux membres cités ci-dessus. Un exposé sur les fouilles de Porsuk a été présenté par Françoise Laroche-Traunecker.

- un colloque a eu lieu les 24 et 25 octobre 2104 à l'Université de Louvain, dans le cadre de la Societas Anatolica : Etat de la recherche sur l'Anatolie antique : Julie Patrier - D. Beyer, "Zeyve HöyükPorsuk : un état des recherches en cours".

- au tout début du mois de juin, l'habituel Symposium archéologique organisé par la Direction générale d'Ankara a eu lieu cette année à Gaziantep. Dominique Beyer y a présenté une communication sur "Zeyve Höyük-Porsuk 2013”.

D. B. et al. 


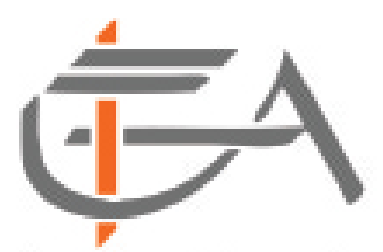

Institut Français d'Etudes Anatoliennes

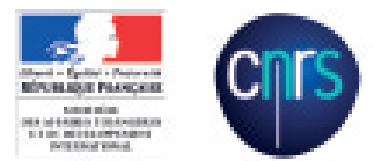

Eglise paléochrétienne de Bindéos (Pisidie), détail de la mosaïque ornant le sal de la nef centrale, $4^{e} \mathrm{~s}$. ap. J.-C. 